\title{
Possible origin for the experimental scarcity of KPZ scaling in non-conserved surface growth
}

\author{
Rodolfo Cuerno ${ }^{\mathrm{a}}$, Mario Castro ${ }^{\mathrm{b}, *}$ \\ ${ }^{a}$ Departamento de Matemáticas \& GISC, Universidad Carlos III de Madrid, \\ Avenida de la Universidad 30, 28911 Leganés, Spain \\ ${ }^{\mathrm{b}}$ Departamento de Sistemas Informáticos, Universidad Pontificia de Comillas, 28008 Madrid, Spain \\ Dedicated to H.E. Stanley on the occasion of his 60 th birthday.
}

\begin{abstract}
The Kardar-Parisi-Zhang (KPZ) equation is generically expected to describe the scaling properties of rough surfaces growing in the absence of conservation laws. However, very few experimental realizations are known of this universality class. Here we focus on the role of instabilities, whether of diffusional origin or other, as physical mechanisms hindering the observation of KPZ scaling. Examples are drawn from various growth processes, such as electrochemical deposition (ECD), chemical vapor deposition (CVD), and erosion by ion-beam sputtering. We moreover consider an interface equation which, starting from the corresponding constitutive equations, can be derived to describe growth by either ECD or CVD depending on the interpretation of the quantities appearing. This approach makes contact with phenomenological parameters, and suggests that a more generic description of non-conserved growth may be provided by the KuramotoSivashinsky equation and generalizations thereof. In this case, the experimental scarcity of KPZ scaling would be due to exceedingly long transients determined by the instabilities that occur.
\end{abstract}

PACS: 68.35.Ct; 64.60.Ht; 81.15.Gh; 81.15.Pq

Keywords: Kinetic roughening; KPZ equation; Kuramoto-Sivashinsky equation; Moving boundary problem; Noise; Ion-beam sputtering; Chemical vapor deposition; Electrochemical deposition

Work partially supported by DGES (Spain) Grant No. BFM2000-0006.

* Corresponding author. Tel.: +34-91-548-44-07; fax: +34-91-541-18-60.

E-mail addresses: cuerno@math.uc3m.es (R. Cuerno), marioc@upco.es (M. Castro).

URLs: http://gisc.uc3m.es/ cuerno, http://gisc.uc3m.es/ mario 


\section{Introduction}

The kinetic roughening of surfaces [1] is a subject of wide interest, due both to its implications for processes of technological relevance [2-4], and to the interesting instances that it offers of extended systems evolving in the presence of fluctuations [5]. A very successful theoretical framework for the study of rough interfaces has been the use of stochastic growth equations for the interface height. Among these, the one proposed by Kardar, Parisi and Zhang (KPZ) [6] has played a prominent role, since in particular it has allowed to make connections to other physical problems, like directed polymers in disordered media or randomly stirred fluids [1]. Denoting by $h(\mathbf{r}, t)$ the surface height at time $t$ above position $\mathbf{r}$ on a reference substrate plane, the KPZ equation is the simplest nonlinear coarse-grained description of a surface growing (or eroding) at a constant average rate $v$ along the local normal direction, and reads

$$
\frac{\partial h}{\partial t}=v+v \nabla^{2} h+\frac{v}{2}(\nabla h)^{2}+\eta(\mathbf{r}, t)
$$

where $v$ is a positive constant, and $\eta(\mathbf{r}, t)$ is an uncorrelated Gaussian noise representing fluctuations, e.g., in a flux of depositing particles. Thus, the KPZ equation was postulated to describe generically the dynamics of surfaces growing in the absence of additional conservation laws, and is thus expected to be relevant to such diverse physical growth systems as erosion by low energy ion-beam sputtering (IBS) [2], electrochemical deposition (ECD) [4] or chemical vapor deposition (CVD) [3]. The generality of KPZ scaling would be a consequence of the phenomenon of universality observed for the scaling properties of rough surfaces.

However, despite some attempts at measuring KPZ scaling in, e.g., IBS [7] or ECD [8], to date very few experiments have been reported which are unambiguously described by the KPZ equation [9-11]. The identification of the physical mechanisms responsible for this paradoxical (termed "anomalous" in some early studies of kinetic roughening) non-KPZ scaling behavior has been complicated by two main reasons: on the one hand, while detailed derivations of the KPZ equation have actually been achieved, they apply to discrete or continuous theoretical models [12] which are indirectly related with experiments, or else the derivations themselves need resort to approximations which are not free from ambiguities. ${ }^{1}$ On the other hand, symmetry arguments such as those leading to the KPZ equation do not enable a detailed connection with phenomenological parameters describing specific experimental systems. These facts have led to invoking additional effects on a coarse-grained level, such as specific noise statistics, non-local effects, etc. [1], in order to account for the difference between the observed and the predicted scaling behaviors of rough surfaces. However, a wide range of scaling exponents ensued, there being no theoretical argument that could identify the correct exponents for a specific growth experiment.

Physically, many of the experimental growth systems expected to be in the KPZ universality class - and which seem to fail such expectations-feature dynamical

${ }^{1}$ This is the case in some works employing the master equation, as discussed in Ref. [13]. 
instabilities leading to development of large slopes on the surface, and even to the production of characteristic surface features whose presence breaks scale invariance. A prototype example is IBS, where standard experimental conditions lead to the production of nanometric ripples [14] or dots [15] on the surface at short and intermediate times. Actually, it has been possible to derive [16] the relevant interface equation for these experiments, which for ions bombarding the target at perpendicular angles reads

$$
\frac{\partial h}{\partial t}=-\left|v_{\mathrm{IBS}}\right|-\left|v_{\mathrm{IBS}}\right| \nabla^{2} h-K_{\mathrm{IBS}} \nabla^{4} h+\frac{\lambda_{\mathrm{IBS}}}{2}(\nabla h)^{2}+\eta(\mathbf{r}, t),
$$

the constants $v_{\mathrm{IBS}}, v_{\mathrm{IBS}}, K_{\mathrm{IBS}}$ and $\lambda_{\mathrm{IBS}}$ being in this case functions of phenomenological quantities, such as the angle of incidence, ion energy, ion penetration depth, etc. Eq. (2) is the noisy generalization of the Kuramoto-Sivashinsky (KS) equation [17]. The KS equation has been seen to describe other dynamic processes such as flame front propagation or chemical turbulence [17], or terrace growth in epitaxial systems [18]. Eq. (2) displays a linear instability at short times, there being a most unstable Fourier mode which induces the observed [14,15] periodic array of ripples or cells. At very long times, scale invariance is restored, the values of the scaling exponents being - at least for a one-dimensional substrate - those of the KPZ universality class [19]. Hence, the specific example of IBS suggests transients due to instabilities as a physical reason for the difficulty in observing the asymptotic KPZ scaling, even if it should occur, since probing asymptotic times may well lie beyond experimental capabilities. For instance, in the CVD experiment in Ref. [11], deposition runs up to 2 days long had to be carried out in order to confirm KPZ behavior, which is both unusual and almost prohibitive. The IBS example illustrates another important fact: trying to assess the origin of non-KPZ behavior implies addressing pre-asymptotic features of the growth process under study. Hence, symmetry arguments do not suffice to derive the interface equation of motion, since contributions which are very important in the early dynamics can be overlooked. For instance, in the IBS system symmetry suggests a simple KPZ description [20], which misses the correct negative sign of the linear Laplacian term in (2) accounting for the physical ripple instability.

\section{Unified equation for CVD and ECD: a stochastic moving boundary problem}

Inspired by the example of IBS, one may wish to reconsider other growth techniques, such as CVD and ECD, for which KPZ scaling is expected to be relevant. These two types of experiments are conceptually similar, their simplest representations [21,22] being essentially as processes in which particles from a vapor (CVD) or solution (ECD) diffuse until they react at an aggregate (CVD) or cathode (ECD) surface, onto which they stick leading to growth. This similarity can be made more precise, to such an extent that the moving boundary problem [23] posed by the system of equations

$$
\begin{aligned}
& \partial_{t} c=D \nabla^{2} c-\nabla \cdot \mathbf{q}, \\
& D \nabla c \cdot \mathbf{n}=\left.k_{D}\left(c-c_{e q}^{0}+\Gamma \kappa+\chi\right)\right|_{s}+\mathbf{q} \cdot \mathbf{n},
\end{aligned}
$$


Table 1

Summary of the most important parameters in model (3)-(6) with their interpretations in CVD and ECD, and some references to precise definitions

\begin{tabular}{lll}
\hline Parameter & CVD & ECD \\
\hline$c$ & Vapor particle concentration & Cation concentration \\
$D$ & Diffusion coefficient & Ambipolar diffusion coefficient [22] \\
$k_{D}$ & Mass transport coefficient & Cationic reaction rate [23] \\
$\Gamma$ & Surface tension coefficient & Idem [23,24] \\
$c_{e q}^{0}$ & Equilibrium concentration at surface & Idem [23,24] \\
$\Omega$ & Molar volume & Idem [23,24] \\
$B$ & Surface diffusion coefficient [21] & Idem [23,24] \\
$c_{a}$ & Particle concentration across stagnant layer & Cation concentration at anode \\
\hline
\end{tabular}

$$
\begin{aligned}
& \mathbf{v} \cdot \mathbf{n}=\Omega(D \nabla c-\mathbf{q}) \cdot \mathbf{n}-B \nabla_{s}^{2} \kappa-\Omega \nabla_{s} \cdot \mathbf{p}, \\
& \lim _{z \rightarrow \infty} c(x, z ; t)=c_{a}
\end{aligned}
$$

can be seen [23] to describe both CVD and ECD growth, as long as the quantities appearing in (3)-(6) are interpreted following Table 1 . In the equations above, $\kappa$ is the surface mean curvature, $\mathbf{n}$ the local surface normal unit vector, $\mathbf{v}$ the aggregate growth velocity, and the subscript $s$ indicates a quantity which is evaluated at the surface. The noise terms $\mathbf{q}, \mathbf{p}$, and $\chi$ represent fluctuations in the diffusion current, the surface diffusion current, and the growth events, respectively [18,23].

Eqs. (3)-(6) are notoriously difficult to solve due to the non-linear coupling between the concentration $c$ and the moving boundary. Notwithstanding, one can obtain perturbatively an equation for the aggregate height $\zeta(x, t)$ in the comoving frame. Details of the calculation can be found in Refs. [23,24]. The result (in Fourier space for the substrate coordinate $x$ ) is

$$
\partial_{t} \zeta_{k}(t)=f(k) \zeta_{k}(t)+\frac{V}{2} N[\zeta]_{k}+\eta_{k}(t),
$$

where $V=k_{D}\left[\Omega\left(c_{a}-c_{e q}^{0}\right)-1\right]$ is the average growth rate, $N[\zeta]_{k}$ is the Fourier transform of $N[\zeta]=\left(\partial_{x} \zeta\right)^{2}$, i.e., the KPZ non-linear term in (1), and $f(k)$ is the linear dispersion relation of Eqs. (3)-(6), which takes different functional forms depending on the values of the mass transfer coefficient $k_{D}$, as seen below. Moreover, $\eta_{k}$ is a noise term induced by the fluctuations $\mathbf{q}, \mathbf{p}$ and $\chi$, with correlations

$$
\left\langle\eta_{k}(t) \eta_{k^{\prime}}\left(t^{\prime}\right)\right\rangle=2 D_{0}(k) \delta\left(k+k^{\prime}\right) \delta\left(t-t^{\prime}\right),
$$

where $D_{0}(k)$ is a function of $k$ whose lowest order contributions account for both conserved and non-conserved noise in (7). Hence we can classify the behavior predicted by Eqs. (3)-(6) according to the relative value of $k_{D}$ (which has velocity units) and the other characteristic velocity of the system, $V$. The coefficient $k_{D}$ depends on the probability that a given diffusing particle sticks to the aggregate. The boundary condition (4) appears (for $\mathbf{q} \equiv \mathbf{0}$ ) in diffusion problems when the arriving particles do not attach deterministically to the aggregate, but rather with a sticking probability $s$. In such a case, it 
can be shown that $[25]$ :

$$
k_{D}=\frac{s}{2-s} D L_{\mathrm{mfp}}^{-1},
$$

where $L_{\mathrm{mfp}}$ is the particle mean free path. Assuming $L_{\mathrm{mfp}}$ is sufficiently small, one has two limits in the above equations: when the sticking probability vanishes then $\nabla c=0$, so the aggregate does not grow. On the contrary, if the sticking probability is close to unity, $k_{D}$ is very large and Eq. (9) reduces to the Gibbs-Thompson relation [26]. In the following we will consider that the capilarity length, $d_{0}$ (which is typically of order $10^{-7}-10^{-6} \mathrm{~cm}$ ) is much smaller than the diffusion length, $l_{D}=D / V \simeq$ $10^{-1}-10^{-2} \mathrm{~cm}$.

\subsection{Non-instantaneous surface kinetics $\left(k_{D} \ll V\right)$}

In this case the dispersion relation is $f(k) \simeq v k^{2}-K k^{4}$, where $v$ and $K$ are related with the system parameters [23]. This dispersion relation is precisely that of the KS equation (2). The unstable dynamics followed by asymptotic KPZ scaling, characteristic of the KS equation, has been actually observed in ECD experiments with Ag deposits performed in Ref. [10]. From the experimental parameters, it can be estimated that $k_{D} \simeq$ $5 \times 10^{-8} \mathrm{~cm} \mathrm{~s}^{-1}$, which is much smaller than $V \simeq 2 \times 10^{-4} \mathrm{~cm} \mathrm{~s}^{-1}$, consistent with the assumption of non-instantaneous surface kinetics. Also, in the CVD experiments in Ref. [11], the low sticking condition is observed to induce asymptotic KPZ scaling, but only after a very long unstable transient.

\subsection{Instantaneous surface kinetics $\left(k_{D} \gg V\right)$}

In this case $f(k) \simeq V|k|-V d_{0} l_{D}|k|^{3}$. This is the well-known Mullins-Sekerka dispersion relation [26], that appears in many other diffusive problems like solidification and, what is more significative, in several ECD experiments like [8,27]. Nevertheless, these experiments were made at constant overpotential instead of constant current density, which is one of the hypothesis made in proposing (3)-(6). A similar experimentbut using a constant current density - was made in Ref. [28], for which the equations above can predict the interface scaling behavior. In this case, the authors observed an initial transient characterized by non-KPZ scaling, followed by unstable growth with a Mullins-Sekerka dispersion relation. Due to a slow growth rate, surface diffusion is relevant in this experiments, hence $f(k)=V|k|\left(1-d_{0} l_{D} k^{2}\right)-B k^{4}$, where $B$ was defined in Table 1. With these parameters, one can integrate numerically Eq. (7) [23,24], to obtain good agreement with the experimental observations.

As mentioned above, $k_{D}$ is related with the sticking probability through Eq. (9). The sticking probability acts as a noise reduction parameter [29] in discrete growth models, as was shown in Ref. [30] for the multiparticle biased diffusion limited aggregation (MBDLA) model, used to study ECD growth. In particular, MBDLA was able to describe quantitatively the morphologies obtained in Ref. [10] as can be seen in Fig. 1. In MBDLA, by reducing the sticking probability the asymptotic KPZ scaling is more 

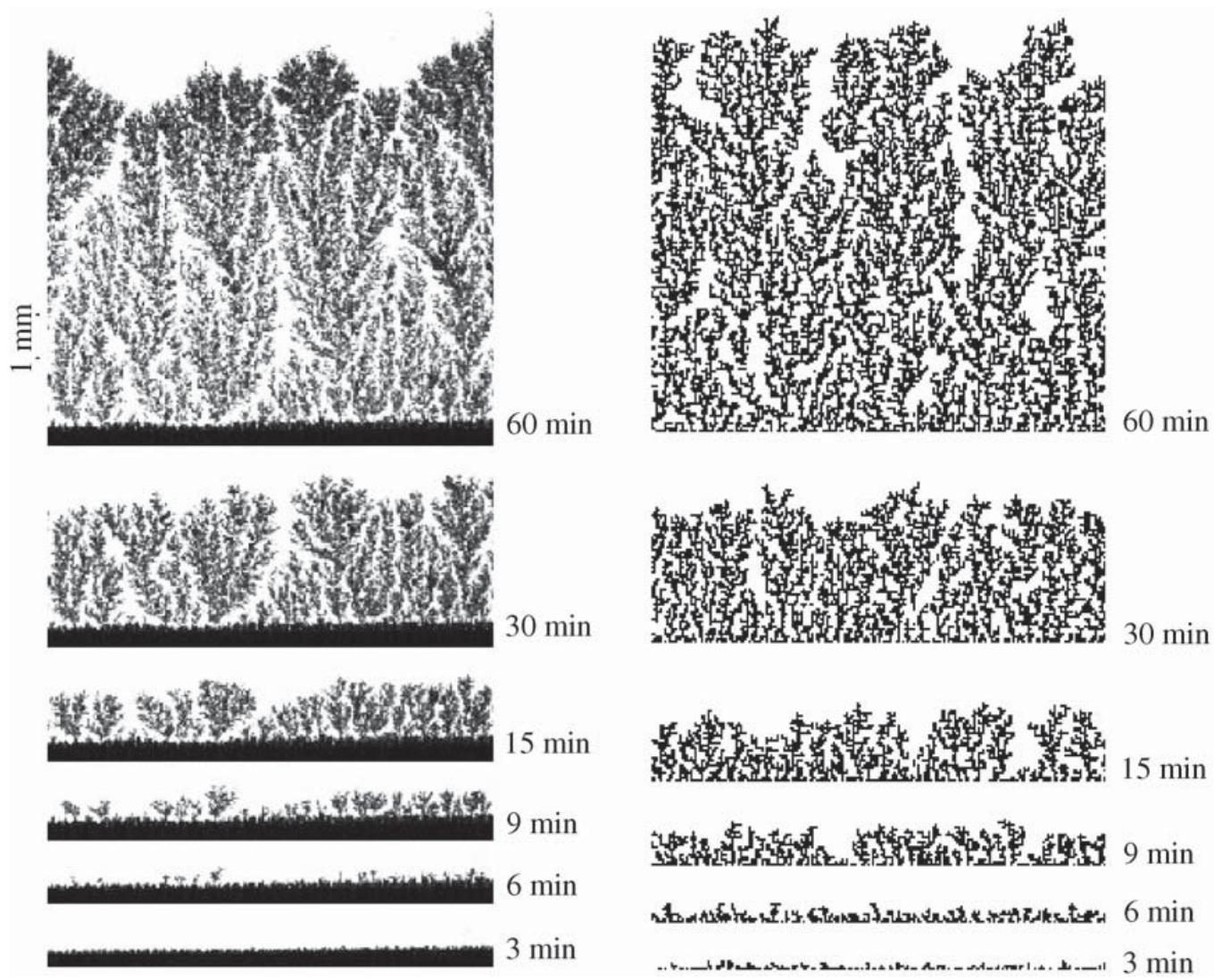

Fig. 1. Left: Electrochemical deposits of Ag from Ref. [10], by kind permission of the authors. Right: MBDLA simulations after Ref. [30].

readily achieved, reducing the importance of pre-asymptotic unstable transients. Hence, noise reduction is not a mere computational tool for discrete models but, rather, it is intimately connected with the surface kinetics via Eqs. (4) and (9).

\section{Conclusions and outlook}

Summarizing, we have illustrated that instabilities appear rather generically in nonconserved surface growth, this occurring as long as the diffusion length $l_{D}$ is larger than the capilarity length $d_{0}$, and independently of the sticking probability at the interface. Nevertheless, the mass transfer coefficient does control both the type of linear dispersion relation and the extent of the transients associated with the instabilities. In deriving a universal equation of motion it then seems that a more accurate-in the sense that more qualitative features are accounted for, like the existence of instabilities - description is provided by the noisy $\mathrm{KS}$ equation and its generalizations (including anisotropy [16], Mullins-Sekerka dispersion relation [23,24]), instead of the KPZ equation. For the case of CVD and ECD, we have seen that connection with phenomenological parameters can be achieved through a moving boundary problem into which fluctuations are incorporated. Moreover, this formulation permits a connection with discrete models 
to be made. Thus, the kinetic mass transfer parameter $k_{D}$ appears as the physical representation of noise reduction parameters.

\section{References}

[1] A.-L. Barabási, H.E. Stanley, Fractal Concepts in Surface Growth, Cambridge University Press, Cambridge, 1995;

J. Krug, Adv. Phys. 46 (1997) 139.

[2] M. Nastasi, J.W. Mayer, J.K. Hirvonen, Ion-Solid Interactions: Fundamentals and Applications, Cambridge University Press, Cambridge, 1996.

[3] K.F. Jensen, W. Kern, in: J.L. Vossen, W. Kern (Eds.), Thin Film Processes II, Academic Press, Boston, 1991.

[4] J.O’M. Bockris, A.K.N. Reddy, Modern Electrochemistry, Plenum/Rosetta, New York, 1970.

[5] J. García-Ojalvo, J.M. Sancho, Noise in Spatially Extended Systems, Springer, New York, 1999.

[6] M. Kardar, G. Parisi, Y.-C. Zhang, Phys. Rev. Lett. 56 (1986) 889.

[7] E.A. Eklund, R. Bruinsma, J. Rudnic, R.S. Williams, Phys. Rev. Lett. 67 (1991) 1759.

[8] G.L.M.K.S. Kahanda, X.-q. Zhou, R. Farrell, P.-z. Wong, Phys. Rev. Lett. 68 (1992) 3741.

[9] R. Paniago, R. Forrest, P.C. Chow, S.C. Moss, S.S.P. Parkin, D. Cookson, Phys. Rev. B 56 (1997) 13 442 ;

J. Maunuksela, M. Myllys, O.-P. Kahkonen, J. Timonen, N. Provatas, M.J. Alava, T. Ala-Nissila, Phys. Rev. Lett. 79 (1997) 1515.

[10] P. Schilardi, O. Azzaroni, R.C. Salvarezza, A.J. Arvia, Phys. Rev. B 59 (1999) 4638.

[11] F. Ojeda, R. Cuerno, R. Salvarezza, L. Vázquez, Phys. Rev. Lett. 84 (2000) 3125.

[12] B. Grossmann, H. Guo, M. Grant, Phys. Rev. A 43 (1991) 1727;

L. Bertini, G. Giacomin, Commun. Math. Phys. 183 (1997) 571.

[13] M. Předota, M. Kotrla, Phys. Rev. E 54 (1996) 3933; an improved approach is in S.-C. Chan, D. Kim, J.-M. Park, Phys. Rev. E 65 (2001) 015102(R).

[14] E. Chason, T.M. Mayer, B.K. Kellerman, D.T. McIlroy, A.J. Howard, Phys. Rev. Lett. 72 (1994) 3040.

[15] S. Facsko, T. Dekorsy, C. Koerdt, C. Trappe, H. Kurz, A. Vogt, H.L. Hartnagel, Science 285 (1999) 1551 ;

R. Gago, L. Vázquez, R. Cuerno, M. Varela, C. Ballesteros, J.M. Albella, Appl. Phys. Lett. 78 (2001) 3316.

[16] R.M. Bradley, J.M.E. Harper, J. Vac. Sci. Technol. A 6 (1988) 2390;

R. Cuerno, A.-L. Barabási, Phys. Rev. Lett. 74 (1995) 4746;

M. Makeev, R. Cuerno, A.-L. Barabási, http://xxx.arxiv.org/abs/cond-mat/0007354.

[17] Y. Kuramoto, T. Tsuzuki, Prog. Theor. Phys. 55 (1976) 356; G.I. Sivashinsky, Acta Astronaut. 6 (1979) 569.

[18] A. Karma, C. Misbah, Phys. Rev. Lett. 71 (1993) 3810;

O. Pierre-Louis, C. Misbah, Phys. Rev. Lett. 76 (1996) 4761;

O. Pierre-Louis, C. Misbah, Phys. Rev. B 58 (1998) 2259;

O. Pierre-Louis, C. Misbah, Phys. Rev. Lett. 58 (1998) 2276.

[19] K. Sneppen, J. Krug, M.H. Jensen, C. Jayaprakash, T. Bohr, Phys. Rev. A 46 (1992) R7351;

R. Cuerno, H.A. Makse, S. Tomassone, S.T. Harrington, H.E. Stanley, Phys. Rev. Lett. 75 (1995) 4464;

S. Park, B. Kahng, H. Jeong, A.-L. Barabási, Phys. Rev. Lett. 83 (1999) 3486.

[20] R. Bruinsma, The KPZ model and sputter erosion, in: R. Jullien, et al., (Eds.), Surface Disordering: Growth, Roughening and Phase Transitions, Nova Science, New York, 1992.

[21] C.H.J. Van den Brekel, A.K. Jansen, J. Cryst. Growth 43 (1977) 364;

B.J. Palmer, R.G. Gordon, Thin Solid Films 158 (1988) 313;

G.S. Bales, A.C. Redfield, A. Zangwill, Phys. Rev. Lett. 62 (1989) 776.

[22] D. Kashchiev, A. Milchev, Thin Solid Films 28 (1975) 189;

J.-N. Chazalviel, Phys. Rev. A 42 (1990) 7355;

S.C. Hill, J.I.D. Alexander, Phys. Rev. E 56 (1997) 4317;

C. Lèger, J. Elezgaray, F. Argoul, Phys. Rev. E 58 (1998) 7700. 
[23] R. Cuerno, M. Castro, Phys. Rev. Lett. 87 (2001) 236 103;

M. Castro, R. Cuerno, to be published.

[24] M. Castro, Ph.D. Thesis, Universidad Complutense de Madrid, 2001 (in Spanish). http://gisc. uc3m.es/ mario/tesis/.

[25] K.R. Naqvi, K.J. Mork, S. Waldenstrøm, Phys. Rev. Lett. 49 (1982) 304.

[26] Y. Saito, Statistical Physics of Crystal Growth, World Scientific, Singapore, 1996.

[27] J.R. de Bruyn, Phys. Rev. E 53 (1996) R5561.

[28] J.M. Pastor, M.A. Rubio, Phys. Rev. Lett. 76 (1996) 1848.

[29] J. Kertész, D.E. Wolf, J. Phys. A 21 (1988) 747.

[30] A. Sánchez, M.J. Bernal, J.M. Riveiro, Phys. Rev. E 50 (1994) R2427;

M. Castro, R. Cuerno, A. Sánchez, F. Domínguez-Adame, Phys. Rev. E 57 (1998) R2491;

M. Castro, R. Cuerno, A. Sánchez, F. Domínguez-Adame, Phys. Rev. E 62 (2000) 161. 